\title{
Associação entre osteoporose e aptidão física de idosos praticantes de exercícios físicos
}

\author{
Giovana Zarpellon Mazo * Janeisa Franck Virtuoso* Inês \\ Alessandra Xavier Lima ${ }^{* \star \star}$ Luana Meneghini ${ }^{* \star * *}$ Maíra Naman ${ }^{\star \star \star \star *}$
}

Resumo: Objetivo - associar o desempenho das aptidões físicas e presença ou não de osteoporose (op) em idosos praticantes de exercícios físicos. Métodos - participaram do estudo 327 idosos (264 mulheres e 63 homens) fisicamente ativos. Aplicou-se uma ficha diagnóstica com dados sociodemográficos e diagnóstico de op, teste de força de preensão manual (fpm) e a bateria senior fitness test. 0 tratamento dos dados deu-se de maneira descritiva (frequência simples e porcentagem) e inferencial (qui-quadrado e mann-whitney). 0 nível de significância de $5 \%$. Resultados - entre os idosos avaliados, $34(12,4 \%)$ relataram ter op. A amostra total apresentou baixo desempenho nas aptidões físicas avaliadas. Associando-se à presença de op, observou-se que os idosos com op têm $\mathrm{fpm}$, força de membros inferiores (mmii) e flexibilidade de membros superiores (mmss) reduzidas quando comparados com idosos sem op. Conclusão - deve-se promover aos idosos com op, exercícios de fortalecimento e alongamento muscular.

Descritores: Osteoporose; Idoso; Aptidão Física.

\section{Association between osteoporosis and fitness for senior practitioners of physical activity}

Abstract: Objective - associate the performance of the physical skills and the presence or not of osteoporosis (op) on seniors who practice physical activity. Methods - 327 senior attendants (264 women and 63 men) physically active. They were applied to a diagnose file with the sociodemographic information and self report of clic diagnostic of op, handgrip strength (fpm) and to senior fitness test battery. The processing of the information happened on a descriptive way (simple frequency and percentage) and inferential (chi-square and mann-whitney test). The level of significance of $5 \%$. Results - between senior evaluated, $34(12, \%)$ reported they have op. Total sample have presented a low performance on the physical abilities evaluated. By associating the presence of op, observed that the seniors with op have reduced: fpm, strength on the superior and flexibility on the inferiors limbs when compared to seniors without op. Concluison - must promote to the seniors with op, exercises of fortification and muscular stretching.

Descriptors: Osteoporosis; Aged; Physical Fitness.

${ }^{*}$ Doutora em Ciências do Desporto pela Universidade do Porto (UP), Porto, Portugal.

**Doutoranda em Ciências do Movimento Humano na Universidade do Estado de Santa Catarina (UDESC), Florianópolis, SC, Brasil.

***Mestre em Educação Física pela Universidade Federal de Santa Catarina (UFSC), Florianópolis, SC, Brasil.

${ }^{* * * *}$ Mestre em Neurociência pela Universidade Federal de Santa Catarina (UFSC), Florianópolis, SC, Brasil.

${ }^{* * * * *}$ Mestranda em Ciências do Movimento Humano na Universidade do Estado de Santa Catarina (UDESC), Florianópolis, SC, Brasil. 


\section{Introdução}

O aumento da taxa das doenças crônico-degenerativas, como a osteoporose, está diretamente relacionado ao envelhecimento da população ${ }^{1}$. No Brasil, o número de pessoas que possuem osteoporose chega a 10 milhões e os gastos com tratamento e assistência no Sistema Único de Saúde (SUS) são altos. Em 2010, gastou-se aproximadamente R\$ 81 milhões para a atenção ao paciente com osteoporose vítima de queda e fratura ${ }^{2}$. 0 desenvolvimento de doenças e a perda progressiva das aptidões funcionais interferem na qualidade de vida do idoso, limitando sua capacidade para realização das atividades da vida diária e prejudicando sua saúde 3 .

Dessa forma, estudos indicam os benefícios da prática de exercício físico regular sobre os níveis de força muscular em idosos ${ }^{4,5}$, equilíbrio ${ }^{5,6}$, redução do risco de quedas6, endurance 4 e capacidade funcional ${ }^{7}$. Além disso, a prática regular de exercícios físicos mantém as atividades ósseas normais, promovendo alterações no metabolismo ósseo por efeito direto via força mecânica ou indireto por fatores hormonais ${ }^{8,9}$.

Apesar desses benefícios, idosos praticantes de exercícios físicos ainda apresentam baixo desempenho em algumas aptidões físicas ${ }^{10}$. Dessa forma, é importante para a prática clínica do fisioterapeuta, conhecer como associam-se o desempenho nas aptidões físicas e a ocorrência de osteoporose entre idosos praticantes de exercícios físicos. A modalidade de exercício físico ideal para balizar o planejamento de propostas de intervenção coerentes com a realidade de idosos acometidos pela osteoporose também é importante conhecer e detectar precocemente o declínio nas aptidões físicas.

Diante disso, o presente estudo tem como objetivo associar o desempenho de diferentes aptidões físicas e a ocorrência de osteoporose em idosos praticantes de exercícios físicos regulares.

\section{Metodologia}

A população desse estudo descritivo foi composta por 400 idosos participantes dos projetos de atividades físicas do Grupo de Estudos da Terceira Idade (GETI) da Universidade do Estado de Santa Catarina (UDESC) e do Programa Saúde e Lazer do Instituto Federal de Santa Catarina (IF/SC). A amostra foi selecionada de forma intencional, tendo-se como critérios de inclusão: serem idosos ( $\geq 60$ anos de idade) e praticantes de exercícios físicos por no mínimo 6 meses. Dessa forma, fizeram parte do estudo 327 idosos de ambos os sexos, sendo 264 mulheres (média de idade de 66,74 $\pm 8,05$ anos) e 63 homens (média de idade de $69,00 \pm 8,88$ anos).

As aulas de exercício físico dos programas têm duração de 50 minutos e são realizadas de duas a três vezes por semana. Essas aulas prezam por uma intensidade moderada em que os idosos precisam de algum esforço físico para realizá-las, bem como, a necessidade de impor uma respiração um pouco mais forte que o normal. As modalidades praticadas pela maioria são: hidroginástica $(38,2 \%)$, natação $(26,9 \%)$, e ginástica $(25,6 \%)$.

O diagnóstico clínico de osteoporose foi identificado segundo o autorrelato dos idosos

Saúde (Santa Maria), v.39, n.2, p.131-140, 2013. Associação entre a osteoporose e aptidão fisica de ISSN 2236-58347 (Você recebeu o diagnóstico de osteoporose de um profissional médico ou de outro profissional da saúde?). Também foram coletados dados referentes às características sociodemográficas. 
Para caracterização da amostra, foi utilizada uma ficha diagnóstica contendo as seguintes informações: idade, sexo, dados sociodemográficos e autorrelato de diagnóstico clínico de osteoporose.

A FPM foi mensurada por meio do teste de força máxima de preensão manual realizado por meio de um dinamômetro - Hydraulic Hand Dynamometer - Modelo Sh5001 - da Saehan Corporation. Para avaliar as demais aptidões físicas, utilizou-se os testes físicos da bateria de testes Senior Fitness Test - SFT11 que é específica para idosos, de fácil aplicação e baixo custo operacional ${ }^{12}$.

Os dados foram coletados por alunos do CEFID/UDESC previamente treinados. As avaliações foram realizadas no local onde eram realizados os exercícios físicos. Primeiramente foi aplicada a ficha diagnóstica em forma de entrevista individual. Em seguida, os testes de aptidão física para idosos Senior Fitness Test - SFT, conforme seu protocolo ${ }^{11}$ que avalia as seguintes aptidões físicas: força e flexibilidade dos membros superiores (MMSS) e inferiores (MMII), resistência aeróbica e equilíbrio dinâmico, e o teste de força máxima de preensão manual, seguindo os procedimentos recomendados por Innes ${ }^{13}$, o qual foi corrigido de acordo com o sexo e índice de massa corporal.

Para obter a classificação do desempenho em cada teste de aptidão física, utilizou-se a escala normal das contagens dos percentis, segundo sexo e faixa etária, conforme proposto por Rikli e Jones ${ }^{11}$. A classificação é dada, para cada teste de aptidão física, em cinco níveis (muito fraco, fraco, regular, bom e muito bom). Para análise, os idosos foram categorizados em "Bom" (Muito Bom e Bom) e "Ruim" (Muito Fraco, Fraco, Regular), para cada teste de aptidão física. Apesar de não ter sido validada para a população idosa brasileira, estudo prévio ${ }^{14}$ encontrou bons resultados com a referida classificação.

Para obter a categorização dos idosos por característica da modalidade de exercício físico realizado, utilizou-se o agrupamento das modalidades disponibilizadas em diferentes grupos (Quadro 1).

Quadro 1 - Categorias das modalidades de exercício físico em diferentes grupos.

\begin{tabular}{cc}
\hline Categoria & Modalidades \\
\hline Grupo 1 & Natação, caminhada, hidroginástica, dança \\
Grupo 2 & Pilates, ginástica, musculação \\
Grupo 3 & Uma modalidade pertencente do grupo 1 ou grupo 2 \\
\hline
\end{tabular}

Os dados foram organizados no programa Microsoft Excel ${ }^{\circledR}$ e analisados no programa estatístico SPSS - Statistical Package for Social Sciences - versão 17.0 para Windows. 0 tratamento estatístico descritivo foi realizado mediante frequência simples e porcentagens. $\mathrm{Na}$ análise inferencial, utilizou-se o teste Qui-Quadrado $\left(X^{2}\right)$ para associar variáveis categóricas ou teste $U$ de Mann-Whitney para comparar variáveis numéricas. Adotou-se um nível de significância de $5 \%$.

Essa pesquisa foi conduzida dentro dos padrões exigidos pela resolução n. ${ }^{0}$ 196/96 do Conselho Nacional de Saúde e aprovada pelo Comitê de Ética em Pesquisas em Seres Humanos da UDESC, sob o protocolo número 185/07.

Rev. Saúde (Santa Maria), Santa Maria, v.39, n.2, p.131-140 Jul./Dez.2013. Mazo, G. Z.; et al. 


\section{Resultados}

Foram entrevistados 327 idosos, sendo 264 mulheres $(80,7 \%)$ e 63 homens (19,3\%). A média de idade dos participantes foi $67,18 \pm 8,25$ anos, havendo diferença significativa ( $U=$ $6881,50 ; p=0,033)$ entre mulheres $(66,74 \pm 8,05$ anos) e homens $(69,00 \pm 8,88$ anos). Quanto às características sociodemográficas da amostra, a maioria é viúvo $(59,8 \%)$, tem ensino fundamental incompleto $(27,9 \%)$, é aposentado/pensionista $(61,1 \%)$ e tem renda mensal familiar acima de 6 salários mínimos (32,8\%).

Com relação à presença de osteoporose, nota-se que $34(12,4 \%)$ dos idosos entrevistados relataram a doença. $\mathrm{Na}$ associação com $\mathrm{O}$ sexo, não houve diferença significativa $\left(X^{2}=3,254 ; p=0,071\right)$, demonstrando que essa doença acomete homens e mulheres de maneira semelhantes, embora com prevalências diferentes $(1,8 \%$ e $10,6 \%$, respectivamente). Já quanto à variável idade, houve diferença significativa ( $U=3769,00 ; p=$ $0,006)$ entre idosos com $(70,73 \pm 7,43)$ e sem $(66,83 \pm 8,24$ anos) osteoporose.

Com relação à prática das modalidades ofertadas, identificou-se que $16(51,6 \%)$ dos idosos que relataram quadro clínico de osteoporose realizam modalidades predominantemente aeróbias, $8(25,8 \%)$ realizam modalidades predominantemente anaeróbias, enquanto $7(22,5 \%)$ realizam modalidades mistas (tanto aeróbias como anaeróbias). $\mathrm{Na}$ associação com os resultados referentes às aptidões físicas, não houve diferença significativa, demonstrando que idosos com osteoporose praticantes de exercícios físicos têm semelhante aptidão física, independentemente do tipo de modalidade que realizam.

Na Tabela 1, apresenta-se a associação/comparação entre presença e ausência de osteoporose com as aptidões físicas dos idosos praticantes de exercícios físicos.

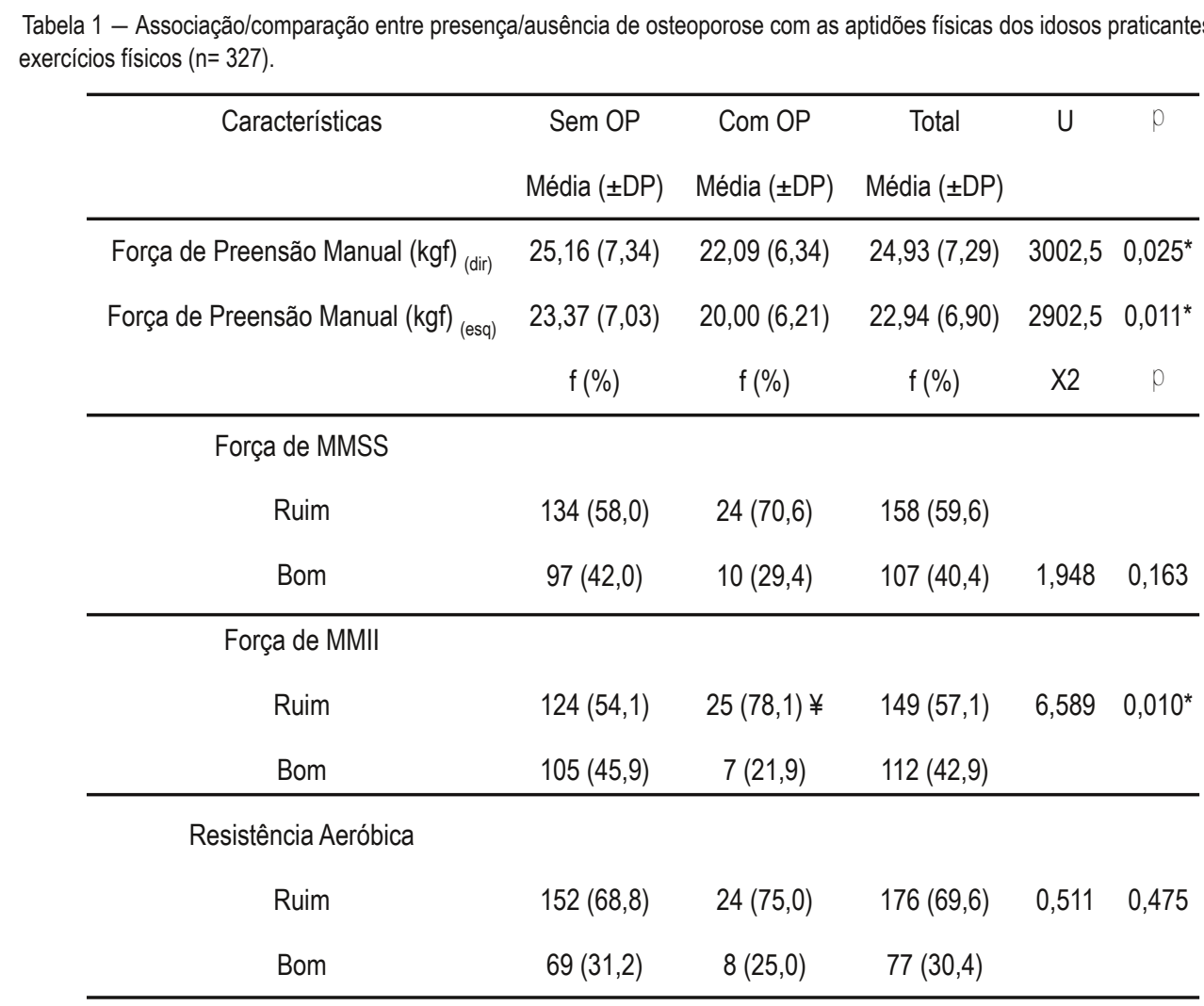

Saúde (Santa Maria), v.39, n.2, p.131-140, 2013. Associação entre a osteoporose e aptidão fisica de idosos praticantes de exercicios físicos 
Flexibilidade de MMII

\begin{tabular}{llllll} 
Ruim & $176(75,2)$ & $23(67,6)$ & $199(74,3)$ & 0,889 & 0,346 \\
Bom & $58(24,8)$ & $11(32,4)$ & $69(25,7)$ & & \\
\hline
\end{tabular}

Flexibilidade de MMSS $_{(\text {dir }}$

\begin{tabular}{cccccc} 
Ruim & $194(83,3)$ & $28(82,4)$ & $222(83,1)$ & 0,17 & 0,895 \\
Bom & $39(16,7)$ & $6(17,6)$ & $45(16,9)$ & \\
\hline
\end{tabular}

Flexibilidade de MMSS $_{(\text {esq })}$

\begin{tabular}{cccccc} 
Ruim & $213(91,4)$ & $27(79,4) ¥$ & $240(89,9)$ & 4,704 & $0,030^{*}$ \\
Bom & $20(8,6)$ & $7(20,6)$ & $27(10,1)$ & \\
\hline
\end{tabular}

Agilidade e equilíbrio dinâmico

\begin{tabular}{|c|c|c|c|c|}
\hline Ruim & $193(83,2)$ & $26(76,5)$ & $219(82,3)$ & 0,920 \\
\hline Bom & $39(16,8)$ & $8(23,5)$ & $47(17,7)$ & \\
\hline
\end{tabular}

$\mathrm{OP}=$ osteoporose; $\mathrm{U}=$ estatística do teste de Mann-Whitney; $\mathrm{f}=$ Freqüência; $\mathrm{X}^{2}=$ estatística do teste Qui-Quadrado; dir= direita; esq= esquerda; MMSS= membros superiores; $M M I I=$ membros inferiores; $p=$ nível de significância * $p \leq 0,05 ; ¥$ ajuste residual $(\geq 2,0)$.

Verifica-se que a FPM, tanto direita quanto esquerda, foi menor entre idosos com osteoporose quando comparada aos idosos sem osteoporose. A FPM apresentou-se reduzida na amostra total (mão direita= 24,93 kgf e mão esquerda= 22,94 kgf).

Observa-se também que, com relação à presença de osteoporose, houve associação com a força dos MMII $\left(X^{2}=6,589 ; p=0,010\right)$ e a flexibilidade do membro superior esquerdo $\left(X^{2}=4,704 ; p=0,030\right)$. Verifica-se que há uma tendência dos idosos com diagnóstico autorrelatado de osteoporose a apresentar pior desempenho na força dos MMII e na flexibilidade do membro superior esquerdo.

As demais aptidões físicas analisadas (flexibilidade do MMII, flexibilidade do membro superior direito, força do membro superior, agilidade e equilíbrio dinâmico e resistência aeróbica) não se associaram com osteoporose. No entanto, observa-se a alta freqüência de idosos com desempenho ruim nessas aptidões.

\section{Discussão}

No presente estudo, a prevalência de osteoporose foi de $12,4 \%$ e associou-se a reduzidas FPM, força de MMII e flexibilidade de MMSS. Além disso, pode-se creditar parte dessas alterações a diferença de idade encontrada entre os grupos com e sem osteoporose. Esses resultados apontam a importância de intervenções pautadas no aumento da força muscular entre a população que envelhece e apresenta diagnóstico de osteoporose.

Alguns estudos $15,16,17$ sugerem que a força muscular corporal global pode ser representada pela FPM, demonstrando correlações significativas entre esta e outras medidas de força de extremidades e de capacidade funcional. Comparando-se as médias encontradas Rev. Saúde (Santa Maria), Santa Maria, v.39, n.2, p.131-140 Jul./Dez.2013. nos idosos do estudo com os valores de referência de Bohannon et al. ${ }^{18}$, observa-se baixa Mazo, G. Z: etal. 
FPM, o que pode interferir nas atividades de vida diária, visto que a baixa FPM indica diminuição de força muscular, sendo esta necessária para a realização de funções básicas como vestir-se e carregar objetos ${ }^{19}$.

A FPM pode ser considerada um preditor de força corporal global e os seus índices abaixo do esperado para faixa etária e sexo podem indicar redução de força de MMII, impactando negativamente na capacidade do indivíduo em efetuar independentemente trocas de postura, marcha, levantar e sentar, subir e descer degraus e manter o equilíbrio ${ }^{20}$. Além disso, baixos valores de FPM podem ser acompanhados por maior tempo de internações hospitalares, maior taxa de mortalidade, aumento dos custos de saúde, necessidade de cuidados de longa duração e maior possibilidade de incapacidade funcional17,18,21,22,23. Esse quadro pode ser ainda mais complicado em idosos com osteoporose devido às características inerentes à patologia.

No presente estudo também foi verificado, nos idosos com osteoporose, um baixo desempenho da aptidão física força dos MMII. Estudos ${ }^{24,25}$ apontam que as perdas tanto de força quanto de massa muscular ocorrem naturalmente com o passar da idade.

Barbosa, Souza e Lebrão ${ }^{26}$ realizaram um estudo com 1656 idosos da cidade de São Paulo com o objetivo de verificar a influência da idade sobre a força e a flexibilidade. Os indivíduos foram distribuídos em três grupos (60-69 anos, 70-79 anos e $\geq$ de 80 anos) e os resultados indicaram que quanto maior a idade, menor é a força e a flexibilidade dos idosos.

Para Guimarães e Farinatti2 ${ }^{27}$, a diminuição de força muscular em MMll é comum nos idosos e tem sido identificada como a segunda maior causa de quedas. A presença de osteoporose torna o problema das quedas ainda maior devido a sobremorbidade nesse grupo.

A prática de exercício físico regular influencia na manutenção das atividades ósseas fisiológicas e vem sendo indicado no tratamento da osteoporose9. Apesar de no presente estudo a modalidade de exercício físico não associar-se ao desempenho das aptidões físicas em idosos com osteoporose, uma revisão sistemática desenvolvida por Santos e Borges ${ }^{28}$ apontou que os exercícios mais estudados no tratamento da osteoporose foram aqueles de extensão isométrica de tronco (em posição antigravitacional), exercícios em cadeia cinética aberta, corridas, caminhadas e exercício de equilíbrio e coordenação.

A manutenção de um estilo de vida ativo retarda os efeitos biológicos negativos do envelhecimento, preservando a autonomia do idoso por meio da melhoria das qualidades físicas da aptidão funcional ${ }^{29}$. Contudo, apesar da prática de exercícios físicos, o processo natural do envelhecimento influencia na queda do desempenho da aptidão funcional ${ }^{30}$. É o que se observa no presente estudo, no qual idosos praticantes de exercício físico apresentam baixo desempenho em algumas aptidões físicas.

Sabe-se que a perda da flexibilidade e da força são as principais variáveis motoras relacionadas às maiores limitações das atividades de vida diária registradas nesta população31. Alterações músculotendíneas afetam especialmente os músculos bi-articulares, limitando a amplitude de movimento e alterando a distribuição do torque em torno das articulações $^{32}$. Gallon et al. ${ }^{33}$ afirmam que exercícios de alongamento podem ser uma importante ferramenta para minimizar estas perdas.

Com relação à flexibilidade, exercícios voltados para o aprimoramento desta aptidão física são normalmente incluídos em qualquer prescrição de exercício físico, tanto para atletas como para indivíduos sedentários e até mesmo para portadores de diversas doenças, e 
particularmente para indivíduos com mais de 65 anos de idade, segundo as recomendações institucionais da American Heart Association e do American College of Sports Medicine, e podem resultar em melhora da qualidade de vida ${ }^{34}$.

\section{Conclusão}

O processo de envelhecimento desencadeia uma série de adaptações nos diferentes sistemas do organismo, que podem prejudicar a aptidão e o desempenho físico quando associadas à falta de exercício físico. Entretanto, no presente estudo os idosos praticantes de exercício físico apresentaram baixo desempenho nas aptidões físicas avaliadas.

Associando-se à presença de osteoporose, observou-se que os idosos com essa patologia tem FPM, força de MMII e flexibilidade de MMSS reduzidas quando comparados aos seus pares sem osteoporose. Estes idosos também apresentaram média de idade maior, o que pode ter influenciado nas aptidões avaliadas.

A maioria dos idosos com esse quadro clínico realizava exercícios físicos predominantemente aeróbios. Diante disso, propõe-se que as intervenções fisioterapêuticas voltadas aos idosos com osteoporose incluam, além do trabalho aeróbio, exercícios de flexibilidade e fortalecimento muscular como forma de minimizar o declínio no desempenho das aptidões físicas avaliadas e amenizar o processo da doença. Da mesma forma, tão importante quanto a prática de exercício físico é a adoção de um estilo de vida ativo, priorizando um envelhecimento saudável.

Sugere-se que novos estudos sejam realizados com uma amostra maior e mais homogênea potencializando, dessa forma, a generalização dos resultados encontrados. Além disso, indica-se que o diagnóstico de osteoporose seja realizado por meio de testes mais apurados, assim como a utilização de baterias validadas na população idosa brasileira.

\section{Referências Bibliográficas}

1. Carvalho JAM, Garcia RAG. O envelhecimento da população brasileira um enfoque demográfico. Cad Saúde Pública. 2003;19(3):725-33.

2. Brasil. Ministério da Saúde. Portal da Saúde. Prevenção á osteoporose deve começar na infância. Brasilia, $\mathrm{DF}, 2010$.

Disponível em: http://portalsaude.saude.gov.br/portalsaude/noticia/2743/162/prevencao-a-osteoporose-devecomecar-na-infancia.html. Acesso em: 14/01/2013.

3. Alves LC, Leimann BCQ, Vasconcelos MEL, Carvalho MS, Vasconcelos AGG, Fonseca, TCO, et al. A influência de das doenças crônicas na capacidade funcional de idosos do Município de São Paulo, Brasil. Cad Saúde Pública. 2007;23(8):1924-30.

4. Rubenstein KR, Laurence ZR, Josephson KR, Trueblood SL, Loy S, Harker JO, et al. Effects of a group exercise program on strength, mobility, and falls among aall-prone elderly men. J Gerontol A Biol Sci Med Sci. 2000;55(6):317-21.

5. Aveiro MC, Navega MT, Granito RN, Rennó ACM, Oishi J. Efeitos de um programa de atividade física no Rev. Saúde (Santa Maria), Santa Maria, v.39, n.2, p.131-140 Jul./Dez.2013. equilibrio e na força muscular do quadríceps em mulheres osteoporóticas visando uma melhoria na qualidade de vida. Rev Bras Ciênc Mov. 2004;12(3):33-8. 
6. Howe TE, Rochester L, Jackson A, Banks PMH, Blair VA. Exercise for improving balance in older people. Cochrane Database Systematic Rev. 2011;11.

7. Barretoi PS. Adesão a longo prazo a um tipo de exercício físico: relação com a capacidade física funcional e motivações pessoais entre mulheres idosas fisicamente independentes. Rev Bras Ciênc Esporte. 2011;33(1):193-206.

8. Henderson NK, Christopher P, White CP, Eisman JA. The roles of exercise and fall risk reduction in the prevention of osteoporosis. Endocrinol Metab Clin North Am. 1998;27(2):369-87.

9. Ocarino NM, Serakides R. Efeito da atividade física no osso normal e na prevenção e tratamento da osteoporose. Rev Bras Med Esporte. 2006;12(3):164-8.

10. Kura GG, Ribeiro LSP, Niquetti R, Tourinho FH. Nível de atividade física, IMC e índices de força muscular estática entre idosas praticantes de Hidroginástica e ginástica. Rev Bras Ciên Envelhecimento Hum. 2004;1(2):30-40.

11. Rikli RE, Jones CJ. Testes de aptidão física para idosos. São Paulo: Manole, 2008.

12. Rikli RE, Jones CJ. Development and validation of a functional fitness test for community-residing older adults. J Aging Phys Activity 1999;7:129-6.

13. Innes A. Handgrip strength testing: a review of literature. Aust Occup Ther J. 1999;46(3):120-40.

14. Streit IA, Mazo GZ, Virtuoso JF, Menezes EC, Gonçalves E. Aptidão física e ocorrência de quedas em idosos praticantes de exercícios físicos. Rev Bras Ativ Fís Saúde. 2011;16(4):346-52.

15. Moreira D, Alvarez RRA, Godoy JR, Cambraia AN. Abordagem sobre preensão palmar utilizando 0 dinamômetro JAMAR囚: uma revisão de literatura. Rev Bras Ci e Mov. 2003;11(2):95-9.

16. Sayers SP, Guralnik JM, Thombs LA, Fielding RA. Effect of Leg Muscle Contraction Velocity on Functional Performance in Older Men and Women. J Am Geriatr Soc. 2005;53(3):467-71.

17. Rolland Y, Cances VL, Cesari M, Vellas B, Pahor M, Grandjean H. Physical performance measures as predictors of mortality in a cohort of community-dwelling older French women. Eur $\mathrm{J}$ Epidemiol. 2006;21(2):113-22.

18. Bohannon RW, Peolsson A, Westropp NM, Desrosiers J, Legman JB. Reference values for adult grip strength measured with a Jamar dynamometer: a descriptive meta-analysis. Physiotherapy. 2006;92(1):11-5.

19. Geraldes AAR, Oliveira ARM, Albuquerque RB, Carvalho JM, Farinatti PTV. A força de preensão manual é boa preditora do desempenho funcional de idosos frágeis: um estudo correlacional múltiplo. Rev Bras Med Esporte. 2008;14(1):12-6.

20. Rantanen T, Masaki K, Foley D, Izmirlian G, White L, Guralnik JM. Grip strength changes over 27 yr in Japanese-American men. J Appl Physiol. 1998;85(1):2047-53.

21. Rantanen T. Muscle strength, disability and mortality. Scand J Med Sci Sports. 2003;13(1):3-8.

22. Rantanen T, Volpato S, Ferrucci L, Fried LP, Guralnik JM. Handgrip strength and cause-specific and total mortality in older disabled women: exploring the mechanism. J Am Geriatr Soc. 2003;51(5):636-41.

23. Fried, L. P.; Ferruci, L.; Darer, J.; Williamson, J.; Anderson, G. Untangling the Concepts of Disability, Frailty, and Comorbidity: Implications for Improved Targeting and Care. J Gerontol A Biol Sci Med Sci. 2004;59(3):255-63.

24. Mayer AP, Lopes WA. A influência do método Pilates na aptidão física de idosas do Município de Guarapuava PR. Voos Rev Polidisciplinar Eletrônica da Faculdade Guairacá. 2011;3(2):82-92.

Saúde (Santa Maria), v.39, n.2, p.131-140, 2013. Associação entre a osteoporose e aptidão fisica de idosos praticantes de exercicios físicos
25. Safons PM, Safons MP, Pereira MM, Rodrigues JF. A. Efeitos do Programa Melhor Idade Brasil Telecom de condicionamento físico sobre a força dos membros inferiores de praticantes idosos. Lecturas Educación Física y Deportes. 2006;98(11). Disponível em: http://www.efdeportes.com/efd98/efeitos.htm. Acesso em: 14/01/2013. 
26. Barbosa AR, Souza JMP, Lebrão ML, Marucci MFN. Relação entre estado nutricional e força de preensão manual em idosos do município de São Paulo, Brasil: dados da pesquisa SABE. Cad Saúde Pública. 2005;21(4):1177-85.

27. Guimaraes JMN, Farinatti PTV. Análise descritiva de variáveis teoricamente associadas ao risco de quedas em mulheres idosas. Rev Bras Med Esporte. 2005;11(5):299-305.

28. Santos MLS, Borges GF. Execício físico no tratamento e prevenção de idosos com osteoporose: uma revisão sistemática. Rev Fisioter Mov. 2010;23(2):289-99.

29. Gobbi S, Villar R, Zago AS. Bases Teórico-Práticas do Condicionamento Físico. Rio de Janeiro: Guanabara; 2005.

30. Hoefelmann CP, Benedetti TRB, Danielle LA, Lopes MA, Mazo GZ, Korn S. Aptidão funcional de mulheres idosas ativas com 80 anos ou mais. Motriz. 2011;17(1):19-25.

31. Jacob Filho W. Atividade física e envelhecimento saudável. Rev Bras Educ Fís Esporte. 2006;20(5):73-7.

32. Samuel D, Rowe PJ. Effect of ageing on isometric strength through joint range at knee and hip joints in three age groups of older adults. Gerontology. 2009;55(6):621-9.

33. Gallon D, Rodacki AL, Hernandez SG, Drabovski B, Outi T, Bittencourt LR, Gomes AR. The effects of stretching on the flexibility, muscle performance and functionality of institutionalized older women. Braz J Med Biol Res. 2011;44(3):229-35.

34. Araujo CGS. Avaliação da flexibilidade: valores normativos do flexiteste dos 5 aos 91 anos de idade. Arq Bras Cardiol. 2008;90(4):280-7.

\section{Giovana Zarpellon Mazo}

Endereço para correspondência - Rua Pascal Simone, 358. Bairro: Coqueiros, CEP: 88080-350, Florianópolis, SC, Brasil.

Currículo Lattes: http://lattes.cnpq.br/3218844421449745

E-mail: giovana.mazo@udesc.br

Recebido em 25 de março de 2013.

Aprovado em de 14 outubro de 2013. 
Saúde (Santa Maria), v.39, n.2, p.131-140, 2013. Associação entre a osteoporose e aptidão fisica de

idosos praticantes de exercícios físicos

140 | ISSN 2236-58347 